(C) 2002 International Press

Adv. Theor. Math. Phys. 6 (2002) 847-871

\title{
Mass Renormalization and Energy \\ Level Shift in Non-Relativistic QED
}

\author{
Christian Hainzl $^{1}$, Robert Seiringer ${ }^{2}$ \\ ${ }^{1}$ Mathematisches Institut, LMU München \\ Theresienstrasse 39, 80333 Munich, Germany \\ hainzl@mathematik.uni-muenchen.de \\ ${ }^{2}$ Department of Physics, Jadwin Hall, Princeton University \\ P.O. Box 708, Princeton, New Jersey 08544, USA \\ rseiring@math.princeton.edu
}

Dedicated to Elliott Lieb on the occasion of his 70th birthday

\begin{abstract}
Using the Pauli-Fierz model of non-relativistic quantum electrodynamics, we calculate the binding energy of an electron in the field of a nucleus of charge $Z$ and in presence of the quantized radiation field. We consider the case of small coupling constant $\alpha$, but fixed $Z \alpha$ and ultraviolet cut-off $\Lambda$. We prove that after renormalizing the mass the binding energy has, to leading order in $\alpha$, a finite limit as $\Lambda$ goes to infinity; i.e., the cut-off can be removed. The expression for the ground state energy shift thus obtained agrees with Bethe's formula for small values of $Z \alpha$, but shows a different behavior for bigger values.
\end{abstract}

\footnotetext{
e-print archive: http://lanl.arXiv.org/abs/math-ph/0205044

${ }^{1}$ Marie Curie Fellow

${ }^{2}$ Erwin Schrödinger Fellow. On leave from Institut für Theoretische Physik, Universität Wien, Boltzmanngasse 5, A-1090 Vienna, Austria
} 


\section{Introduction}

Quantum electrodynamics (QED) has proved one of the most successful theories in physics. One of its striking features was the explanation of the so-called Lamb-shift, the splitting of the energy levels of the hydrogen atom. Most of the results, however, are of perturbative nature, and very little is known concerning rigorous results starting from a well-defined (Hamiltonian) theory.

A few years ago, Bach, Fröhlich and Sigal initiated a rigorous non-perturbative study of non-relativistic QED. They made a detailed spectral analysis of the Pauli-Fierz Hamiltonian, which is a model appropriate for studying the low energy properties of QED, and which is the model we use in this paper. Among other results, they proved in [BFS] the existence of a ground state for small values of the coupling constant $\alpha$ and the ultraviolet (UV) cut-off $\Lambda$, which has to be introduced in the interaction term between the electrons and the photon field in order to obtain a well-defined Hamiltonian. Recently, Griesemer, Lieb and Loss [GLL] succeeded in removing these restrictions on the parameters. We refer to [GLL] for an extensive list of references on the subject.

Our goal in this paper is to calculate the binding energy of one electron in the field of a nucleus of charge $Z$. In contrast to the case of a Schrödinger operator without coupling to the quantized photon field, the "bare mass" appearing in the Hamiltonian is not equal to the physical mass. To obtain the binding energy as a function of the physical mass, which can be measured in experiments, and hence to compare the calculated binding energy with the measured one, one has to substitute the physical mass for the bare mass. To do this, one first has to calculate the physical mass as a function of the bare mass, the coupling constant $\alpha$, and the ultraviolet cut-off $\Lambda$. We do this via the dispersion relation, i.e., the energy of a free electron as a function of the total momentum (= electron momentum + field momentum).

There are three parameters in the problem: the coupling constant $\alpha$, the UV cut-off $\Lambda$, and the nuclear charge $Z$. We consider the case when $\alpha$ is small, but $Z \alpha$ not necessarily small. I.e., we first fix $\Lambda$ and $Z \alpha$ and consider $\alpha$ as a small parameter.

Our calculations are to leading order in $\alpha$. We give rigorous error estimates, but do not focus on optimizing the error terms. In particular, we do not have sufficient control on their behavior for large $\Lambda$ to be able to prove the finiteness of the result after removing the cut-off beyond leading order in $\alpha$. This remains an interesting open problem. Steps in this direction were 
taken in [LL1], where the behavior of the self energy for large $\Lambda$ was studied. Bounds on mass renormalization and binding energies were recently given in [LL2].

We shall rigorously show that after renormalizing the mass the UV cutoff can be removed, at least to leading order in $\alpha$. More precisely, the binding energy, as function of the physical mass and the coupling parameter $\alpha$, has, to leading order in $\alpha$, a finite limit as $\Lambda \rightarrow \infty$. We thus obtain an expression for the ground state energy shift due to the presence of the radiation field, which holds to leading order in $\alpha$, and for all values of $Z \alpha$. It agrees with Bethe's formula [Be] for small values of $Z \alpha$, but shows a different behavior for bigger values. We emphasize again that in our formula for the binding energy no UV cut-off appears, in contrast to Bethe's formula, who used the dipole approximation for it's derivation and neglects the spin of the electron. In Bethe's formula a logarithmic dependence on $\Lambda$ appears. Bethe argues that a physically reasonable value for $\Lambda$ is $m c^{2}$, the energy needed for pair production of electrons and positrons to take place. By inserting this value for $\Lambda$ in his formula he obtains excellent agreement with experiments, at least for the case $Z=1$, i.e., the hydrogen atom.

Shortly after Bethe's work, it was noted in [KL] that the Lamb shift ought to be finite after removing the cut-off $\Lambda$ if one does not use the dipole approximation (see also $[\mathrm{AF}]$ ). By means of perturbation theory the Lamb shift was calculated, for both $\alpha$ and $Z \alpha$ small, in [Gr].

Our proofs below essentially show that second order perturbation theory is correct. I.e., to obtain results up to order $\alpha$ only the addition of one photon is needed. We use and extend previous ideas in $[\mathrm{H}]$, where results on the leading order of the self energy and the binding energy were obtained. (Concerning the question of enhanced binding, see [HVV].)

The paper is organized as follows. In the next section, we describe the precise setting and introduce some notation that will be used throughout the paper. In Section 3 we calculate the dispersion relation and thus obtain an expression for the physical mass in terms of the bare mass, i.e., for the necessary mass renormalization. The binding energy is calculated in Section 4. In Section 5 it is shown that after renormalization of the mass, the UV cut-off can be removed, and a finite expression for the binding energy to leading order in $\alpha$ is obtained. See Theorem 4 on page 867 below for the precise statement. Finally we comment on the Lamb shift of metastable excited states in Section 6. 


\section{Setting and Notation}

We now describe the precise setting and introduce useful notations. The Hamiltonian under consideration is the Pauli-Fierz Hamiltonian

$$
H=\frac{1}{2 m_{0}}\left[(p+\sqrt{\alpha} A(x))^{2}+\sqrt{\alpha} \sigma \cdot B(x)\right]+V(x)+H_{f} .
$$

It acts on $\mathcal{H}=L^{2}\left(\mathbb{R}^{3}, d^{3} x ; \mathbb{C}^{2}\right) \otimes \mathcal{F}_{b}\left(L^{2}\left(\mathbb{R}^{3}, d^{3} k ; \mathbb{C}^{2}\right)\right)$, where $\mathcal{F}_{b}$ denotes the bosonic Fock space. Self-adjointness of $H$ on an appropriate domain has recently been shown in [Hi].

Units are chosen such that $\hbar=c=1$. The electron charge is then given by $e=\sqrt{\alpha}$, with $\alpha$ the fine structure constant. In nature $\alpha \approx 1 / 137$, but we consider it here as an arbitrary, small number. The positive parameter $m_{0}$ is the bare mass of the electron.

The $\cdot$ product stands for the usual scalar product in $\mathbb{R}^{3}$, and $\sigma$ denotes the vector of Pauli matrices, acting on the spin variable of the electron part of the wave function. The operator $p=-i \nabla_{x}$ is the electron momentum. The vector potential in Coulomb gauge is

$$
A(x)=\frac{1}{2 \pi} \sum_{\lambda=1,2} \int_{|k| \leq \Lambda} \frac{1}{\sqrt{|k|}} \varepsilon_{\lambda}(k)\left[a_{\lambda}(k) e^{i k \cdot x}+a_{\lambda}^{*}(k) e^{-i k \cdot x}\right] d^{3} k
$$

Here the integration is restricted to momenta $|k| \leq \Lambda$, i.e., we make a sharp UV cut-off. We could allow for a more general, spherically symmetric cut-off, but we restrict ourselves to this case for simplicity. The vectors $\varepsilon_{\lambda}(k) \in \mathbb{R}^{3}$ are any two possible orthonormal polarization vectors perpendicular to $k$. The corresponding magnetic field $B=\operatorname{curl} A$ reads

$$
B(x)=\frac{1}{2 \pi} \sum_{\lambda=1,2} \int_{|k| \leq \Lambda} \frac{i}{\sqrt{|k|}} k \wedge \varepsilon_{\lambda}(k)\left[a_{\lambda}(k) e^{i k \cdot x}-a_{\lambda}^{*}(k) e^{-i k \cdot x}\right] d^{3} k .
$$

The operators $a_{\lambda}^{*}(k)$ and $a_{\lambda}(k)$ are the usual creation and annihilation operators for a photon of momentum $k$ and polarization $\lambda$. They satisfy the commutation relations

$$
\left[a_{\nu}(k), a_{\lambda}^{*}(q)\right]=\delta(k-q) \delta_{\nu \lambda}
$$

and

$$
\left[a_{\nu}^{*}(k), a_{\lambda}^{*}(q)\right]=0, \quad\left[a_{\nu}(k), a_{\lambda}(q)\right]=0
$$


The field energy is given by $H_{f}=d \Gamma(|k|)$, where we denote, in general, the second quantized version of a function $h(k)$ by

$$
d \Gamma(h(k))=\sum_{\lambda=1,2} \int_{\mathbb{R}^{3}} h(k) a_{\lambda}^{*}(k) a_{\lambda}(k) d^{3} k .
$$

A general wave function $\Psi \in \mathcal{H}$ can be written as a sequence

$$
\Psi=\left\{\psi_{0}, \psi_{1}, \psi_{2}, \ldots\right\},
$$

with $\psi_{n}=\psi_{n}\left(x, k_{1}, \ldots, k_{n}\right)$ an $n$-photon wave function. We suppress the dependence on the spin of the electron and the polarization of the photons for simplicity of notation. The creation parts of the vector fields $A$ and $B$ create a photon with wave function $G(k) e^{-i k \cdot x}$ and $H(k) e^{-i k \cdot x}$, respectively. Here $G(k)=\left(G^{1}(k), G^{2}(k)\right)$ and $H(k)=\left(H^{1}(k), H^{2}(k)\right)$ are vectors of onephoton states, given by

$$
G^{\lambda}(k)=\frac{\theta(\Lambda-|k|)}{2 \pi|k|^{1 / 2}} \varepsilon_{\lambda}(k)
$$

and

$$
H^{\lambda}(k)=\frac{-i \theta(\Lambda-|k|)}{2 \pi|k|^{1 / 2}} k \wedge \varepsilon_{\lambda}(k) .
$$

Their vector components will be denoted by $H_{i}$ and $G_{i}$, respectively ( $i=$ $1,2,3)$, and likewise generally for vectors in $\mathbb{R}^{3}$. The function $\theta$ denotes the Heaviside step function, taking the ultraviolet cutoff $\Lambda$ into account.

\section{Dispersion Relation}

We first consider the case of a free electron, i.e., $V=0$. The Hamiltonian is then translation invariant, i.e., it commutes with the three components of the total momentum $P=p+d \Gamma(k)$. It is therefore possible to write the Hilbert space and the Hamiltonian as a direct integral

$$
\mathcal{H}=\int_{\mathbb{R}^{3}}^{\oplus} d^{3} P \mathcal{H}_{P}
$$

and

$$
H=\int_{\mathbb{R}^{3}}^{\oplus} d^{3} P H_{P},
$$


with $H_{P}$ acting on $\mathcal{H}_{P}$. Each $\mathcal{H}_{P}$ is isomorphic to $\mathbb{C}^{2} \otimes \mathcal{F}_{b}$. The Hamiltonian $H_{P}$ in this representation is given by

$$
H_{P}=\frac{1}{2 m_{0}}\left[(P-d \Gamma(k)+\sqrt{\alpha} A(0))^{2}+\sqrt{\alpha} \sigma \cdot B(0)\right]+H_{f} .
$$

For any fixed total momentum $P$, we define $\mathcal{E}_{P}$ to be the ground state energy of $H_{P}$. The existence of a corresponding ground state is of no concern to us. In $[C]$ it was shown, at least for the spinless case, that there does not exist a ground state of $H_{P}$ for $P \neq 0$ unless one imposes an infrared cut-off on $H_{P}$.

The function $\mathcal{E}_{P}$ is often referred to as the dispersion relation. For small $P, \mathcal{E}_{P} \approx \mathcal{E}_{0}+|P|^{2} / 2 m$, where $m$ is, by definition, the physical mass. It is strictly bigger than the bare mass $m_{0}$. For given $\Lambda, m_{0}$ has to be chosen such that $m$ is equal to the mass of the electron, which is determined by experiment. The bare mass goes to zero as the UV cut-off $\Lambda$ goes to infinity, which is usually referred to as renormalization of $m_{0}$.

We now calculate this mass renormalization to leading order in $\alpha$. We restrict ourselves to the case $P<m_{0}$, since we are only interested in the behavior of $\mathcal{E}_{P}$ for small $P$. For large $P$ the behavior is different due to the different energy-momentum relations of the relativistic photon and the non-relativistic electron.

THEOREM 1 (Dispersion Relation). For $|P|<m_{0}$, the energy $\mathcal{E}_{P}$ of a free particle with total momentum $P$ satisfies

$$
\begin{aligned}
\mathcal{E}_{P}-\mathcal{E}_{0}= & \frac{|P|^{2}}{2 m_{0}}-\frac{\alpha}{2 \pi^{2} m_{0}} \int_{|k| \leq \frac{\Lambda}{2 m_{0}}} d^{3} k \frac{|k|}{|k|^{2}+|k|-P \cdot k / m_{0}} \times \\
& \times\left(\frac{|P|^{2}}{|k|^{2}}+|P \cdot k|^{2}\left(\frac{2}{\left(|k|^{2}+|k|\right)^{2}}-\frac{1}{|k|^{4}}\right)\right)+O\left(\alpha^{3 / 2}\right) .
\end{aligned}
$$

Strictly speaking, we do not claim that the error term is truly $O\left(\alpha^{3 / 2}\right)$, but only that it is bounded by const. $\alpha^{3 / 2}$ for small $\alpha$. The same remark applies to Theorems 2 and 3 below.

Proof. Throughout the proof we will set $m_{0}=\frac{1}{2}$ for simplicity. The correct dependence on $m_{0}$ is easily obtained by dimensional analysis. We will provide appropriate upper and lower bounds on $\mathcal{E}_{P}$.

We start with the upper bound: We choose as a trial state

$$
\Psi=\left\{\uparrow, \frac{-\sqrt{\alpha}}{|k|^{2}+|k|-2 P \cdot k+\alpha}[2 P \cdot G(k)+\sigma \cdot H(k)] \uparrow, 0,0, \ldots\right\},
$$


where $G(k)$ and $H(k)$ are defined in (2.8) and (2.9), and where $\uparrow$ is a shorthand notation for the vector $(1,0)$ in spin space. The additional factor $\alpha$ in the denominator of the one-photon part serves as an infrared cut-off to make the norm of $\Psi$ finite.

We write $A=A(0)=D+D^{*}$, where $D$ denotes the part of $A$ containing only annihilation operators. A straightforward calculation, using that

$$
A^{2}=\left(D+D^{*}\right)^{2}=\frac{1}{\pi} \Lambda^{2}+2 D^{*} \cdot D+D^{*} \cdot D^{*}+D \cdot D
$$

and $k \cdot D^{*} \uparrow=k \cdot G(k) \uparrow=0$, yields

$$
\begin{gathered}
\left\langle\Psi\left|H_{P}\right| \Psi\right\rangle=\left(|P|^{2}+\frac{\alpha}{\pi} \Lambda^{2}\right)\langle\Psi \mid \Psi\rangle \\
-\alpha\left\langle(2 P \cdot G+\sigma \cdot H) \uparrow\left|\frac{1}{|k|^{2}+|k|-2 P \cdot k+\alpha}\right|(2 P \cdot G+\sigma \cdot H) \uparrow\right\rangle \\
+\alpha^{2}(\langle\Psi \mid \Psi\rangle-1)+\text { const. } \alpha^{2} .
\end{gathered}
$$

Using the fact the $G$ is real and $H$ is purely imaginary, and the anticommutation relations for $\sigma$, namely

$$
\sigma_{i} \sigma_{j}+\sigma_{j} \sigma_{i}=2 \delta_{i j} I_{\mathbb{C}^{2}}
$$

we get

$$
\begin{array}{r}
\left\langle(2 P \cdot G+\sigma \cdot H) \uparrow\left|\frac{1}{|k|^{2}+|k|-2 P \cdot k+\alpha}\right|(2 P \cdot G+\sigma \cdot H) \uparrow\right\rangle= \\
\sum_{\lambda=1}^{2} \int d^{3} k \frac{4\left|P \cdot G^{\lambda}(k)\right|^{2}+\left|H^{\lambda}(k)\right|^{2}}{|k|^{2}+|k|-2 P \cdot k+\alpha} .
\end{array}
$$

We now insert the expressions (2.8) and (2.9) for $G$ and $H$ and use that $\sum_{\lambda=1}^{2}\left|P \cdot \varepsilon_{\lambda}(k)\right|^{2}=|P|^{2}-|P \cdot k|^{2} /|k|^{2}$ by the orthogonality relations of $k$ and $\varepsilon_{\lambda}$. Moreover, since $\langle\Psi \mid \Psi\rangle=1+O(\alpha \ln (1 / \alpha))$, we arrive at the upper bound

$$
\begin{aligned}
\left.\mathcal{E}_{P} \leq|P|^{2}+\frac{\alpha}{\pi} \Lambda^{2}-\frac{\alpha}{(2 \pi)^{2}} \int_{|k| \leq \Lambda} d^{3} k \frac{4\left(|P|^{2}|k|^{2}-|P \cdot k|^{2}\right)+2|k|^{4}}{|k|^{3}\left(|k|^{2}\right.}+|k|-2 P \cdot k\right) & \\
& +O\left(\alpha^{2} \ln (1 / \alpha)\right) .
\end{aligned}
$$

Lower Bound: We first show that, for suitable constants $C_{i}>0$,

$$
H_{P} \geq\left(|P|^{2}+|d \Gamma(k)|^{2}\right)\left(1-C_{1} \alpha\right)+C_{2} H_{f}-C_{3} \alpha .
$$


Together with the upper bound (3.10) this shows that, in an approximate ground state $\Psi_{0},\left\langle\Psi_{0}\left|H_{f}\right| \Psi_{0}\right\rangle \leq$ const. $\alpha$ and $\left\langle\left.\Psi_{0}|| d \Gamma(k)\right|^{2} \mid \Psi_{0}\right\rangle \leq$ const. $\alpha$.

The fact that $\varepsilon_{\lambda}(k) \cdot k=0$ implies that $d \Gamma(k) \cdot A=A \cdot d \Gamma(k)$. Using this we can estimate by Schwarz' inequality

$$
\begin{aligned}
\pm \sqrt{\alpha}\langle\psi|(P-d \Gamma(k)) \cdot A| \psi\rangle & = \pm 2 \sqrt{\alpha} \Re\langle\psi|(P-d \Gamma(k)) \cdot D| \psi\rangle \\
\leq & \alpha a\left\langle\psi|| P-\left.d \Gamma(k)\right|^{2} \mid \psi\right\rangle+\frac{1}{a}\left\langle\psi\left|D^{*} D\right| \psi\right\rangle
\end{aligned}
$$

for any positive constant $a$. This leads to the operator inequality

$$
\pm \sqrt{\alpha}(P-d \Gamma(k)) \cdot A \leq a \alpha|P-d \Gamma(k)|^{2}+\frac{1}{a} D^{*} D .
$$

In the same manner, we obtain

$$
\pm \sqrt{\alpha} \sigma \cdot B \leq \frac{1}{a^{\prime}} E^{*} E+\alpha a^{\prime}
$$

for a positive $a^{\prime}$. Moreover, $D^{*} D \leq \frac{2}{\pi} \Lambda H_{f}$ and $E^{*} E \leq \frac{2}{3 \pi} \Lambda^{3} H_{f}$ (see, e.g., [GLL, Lemma A.4]; note the different prefactors due to the missing $\frac{1}{2 \pi}$ in their definition of $A$ ). Since $A^{2} \geq 0$ we get

$$
\begin{aligned}
& H_{P} \geq|P-d \Gamma(k)|^{2}(1-2 a \alpha)+H_{f}\left(1-\frac{4}{a \pi} \Lambda-\frac{2}{3 a^{\prime} \pi} \Lambda^{3}\right)-\alpha a^{\prime} \\
& \geq\left(|P|^{2}+|d \Gamma(k)|^{2}\right)(1-2 a \alpha)+H_{f}\left(1-\frac{4}{a \pi} \Lambda-\frac{2}{3 a^{\prime} \pi} \Lambda^{3}-2|P|\right)-\alpha a^{\prime},
\end{aligned}
$$

where the last inequality holds for $\alpha \leq 1 /(2 a)$. Since $|P|<\frac{1}{2}$ by assumption, we can choose the constants $a$ and $a^{\prime}$ appropriately and therefore arrive at (3.11).

Using the results obtained above, we can now estimate $\left\langle\Psi_{0}\left|H_{P}\right| \Psi_{0}\right\rangle$, for an approximate ground state $\Psi_{0}$, from below. By Schwarz' inequality

$$
D^{2}+D^{* 2} \geq-a D^{*} D-\frac{1}{a} D D^{*}
$$

for any $a>0$. Choosing $a=1 / \sqrt{\alpha}>\frac{1}{2}$ we get, using $D D^{*}=D^{*} D+\frac{1}{\pi} \Lambda^{2}$,

$$
D^{2}+D^{* 2}+2 D^{*} D \geq-\frac{1}{\sqrt{\alpha}} D^{*} D-\frac{\sqrt{\alpha}}{\pi} \Lambda^{2} .
$$

Together with $D^{*} D \leq \frac{2}{\pi} \Lambda H_{f}$ and the a priori estimate $\left\langle\Psi_{0}\left|H_{f}\right| \Psi_{0}\right\rangle \leq$ const. $\alpha$ this allows us to conclude that

$$
\left\langle\Psi_{0}\left|\alpha A^{2}\right| \Psi_{0}\right\rangle \geq \frac{\alpha}{\pi} \Lambda^{2}-\text { const. } \alpha^{3 / 2} .
$$


By similar arguments, using Schwarz' inequality as in (3.12), with $a=1 / \sqrt{\alpha}$, and $\left\langle\left.\Psi_{0}|| d \Gamma(k)\right|^{2} \mid \Psi_{0}\right\rangle \leq$ const. $\alpha$,

$$
\left|\left\langle\Psi_{0}|\sqrt{\alpha} d \Gamma(k) \cdot A| \Psi_{0}\right\rangle\right| \leq \text { const. } \alpha^{3 / 2} .
$$

We therefore obtain

$$
\begin{aligned}
& \left\langle\Psi_{0}\left|H_{P}\right| \Psi_{0}\right\rangle \geq|P|^{2}+\frac{\alpha}{\pi} \Lambda^{2}-\text { const. } \alpha^{3 / 2}+ \\
& \left\langle\left.\Psi_{0}|| d \Gamma(k)\right|^{2}-2 P \cdot d \Gamma(k)+2 \sqrt{\alpha} P \cdot A+\sqrt{\alpha} \sigma \cdot B+H_{f}+\alpha^{3 / 2} \mid \Psi_{0}\right\rangle,
\end{aligned}
$$

where we inserted the last term $\alpha^{3 / 2}$ because it will be convenient later. The expression in the second line can be rewritten as

$$
\begin{aligned}
\sum_{n \geq 1}\left[\left\langle\left.\psi_{n}|| d \Gamma(k)\right|^{2}+H_{f}-\right.\right. & 2 P \cdot d \Gamma(k)+\alpha^{3 / 2}\left|\psi_{n}\right\rangle \\
& \left.+2 \sqrt{\alpha} \Re\left\langle\psi_{n}\left|2 P \cdot D^{*}+\sigma \cdot E^{*}\right| \psi_{n-1}\right\rangle\right],
\end{aligned}
$$

where $\psi_{n}$ denotes the $n$-photon part of the wave function $\Psi_{0}$. We introduce the operator

$$
\mathcal{Q}=|d \Gamma(k)|^{2}+H_{f}-2 P \cdot d \Gamma(k)+\alpha^{3 / 2},
$$

which is positive for $|P|<\frac{1}{2}$. By using Schwarz' inequality, we can estimate (3.21) by

$$
(3.21) \geq \sum_{n \geq 1}-\alpha\left\langle\psi_{n-1}\left|(2 P \cdot D+\sigma \cdot E) \frac{1}{\mathcal{Q}}\left(2 P \cdot D^{*}+\sigma \cdot E^{*}\right)\right| \psi_{n-1}\right\rangle .
$$

We now investigate this term. The operator $\left(2 P \cdot D^{*}+\sigma \cdot E^{*}\right)$ acting on $\psi_{n-1}$ creates a photon. Explicitly, in momentum representation, suppressing the polarization in the notation,

$$
\left[E^{*} \psi_{n-1}\right]\left(k_{1}, \ldots k_{n}\right)=\frac{1}{\sqrt{n}} \sum_{i=1}^{n} H\left(k_{i}\right) \psi_{n-1}\left(k_{1}, \ldots, \not_{i}, \ldots, k_{n}\right),
$$

where $h_{i}$ means that $k_{i}$ is omitted from consideration. An analogous expression holds for $D^{*}$. The right side of (3.23) can therefore be split into two parts, one coming from the "diagonal terms", where both on the left and on the right side the photon is created at the $i$ 'th position, and the mixed terms. More precisely, denoting $F(k)=2 P \cdot G(k) I_{\mathbb{C}^{2}}+\sigma \cdot H(k)$ and using permutation symmetry,

$$
(3.23)=-\alpha \sum_{n \geq 1}\left(I_{n}+I I_{n}\right)
$$


where the two terms $I_{n}$ and $I I_{n}$ are given by

$$
I_{n}=\left\langle F\left(k_{n}\right) \psi_{n-1}\left(k_{1}, \ldots, k_{n-1}\right)\left|\frac{1}{\mathcal{Q}}\right| F\left(k_{n}\right) \psi_{n-1}\left(k_{1}, \ldots, k_{n-1}\right)\right\rangle
$$

and

$$
I I_{n}=(n-1)\left\langle F\left(k_{n}\right) \psi_{n-1}\left(k_{1}, \ldots, k_{n-1}\right)\left|\frac{1}{\mathcal{Q}}\right| F\left(k_{1}\right) \psi_{n-1}\left(k_{2}, \ldots, k_{n}\right)\right\rangle .
$$

To estimate $I_{n}$ from above, we write $\mathcal{Q}=\mathcal{A}+b$, with $\mathcal{A}=\left|k_{n}\right|^{2}+\left|k_{n}\right|-$ $2 P \cdot k_{n}$, and use

$$
\frac{1}{\mathcal{A}+b}=\frac{1}{\mathcal{A}}-\frac{b}{\mathcal{A}(\mathcal{A}+b)}
$$

and the estimates $b \geq-2\left|k_{n}\right| \sum_{i=1}^{n-1}\left|k_{i}\right|, \mathcal{A} \geq\left|k_{n}\right|(1-2|P|)$ and $\mathcal{A}+b \geq$ $\left|k_{n}\right|(1-2|P|)$ on the last term. Writing $\psi_{n-1}=\psi_{n-1}\left(k_{1}, \ldots, k_{n-1}\right)$ and

$$
W=W\left(k_{1}, \ldots, k_{n}\right)=\frac{1}{\left|k_{n}\right|^{2}+\left|k_{n}\right|-2 P \cdot k_{n}}+\frac{2\left|k_{n}\right| \sum_{i=1}^{n-1}\left|k_{i}\right|}{\left|k_{n}\right|^{2}(1-2|P|)^{2}}
$$

for short, we get

$$
\begin{aligned}
& I_{n} \leq\left\langle F\left(k_{n}\right) \psi_{n-1}|W| F\left(k_{n}\right) \psi_{n-1}\right\rangle \\
& =4\left\langle P \cdot G\left(k_{n}\right) \psi_{n-1}|W| P \cdot G\left(k_{n}\right) \psi_{n-1}\right\rangle \\
& \quad+\sum_{j=1}^{3}\left\langle H_{j}\left(k_{n}\right) \psi_{n-1}|W| H_{j}\left(k_{n}\right) \psi_{n-1}\right\rangle,
\end{aligned}
$$

where we used again the anti-commutation relations of the Pauli matrices and the fact that the mixed terms vanish, as explained in the upper bound. Inserting the expressions (2.8) and (2.9) for $G(k)$ and $H(k)$ yields (compare with (3.9)-(3.10))

$$
\begin{aligned}
& I_{n} \leq\left\|\psi_{n-1}\right\|^{2} \frac{1}{(2 \pi)^{2}} \int_{|k| \leq \Lambda} d^{3} k \frac{4\left(|P|^{2}|k|^{2}-|P \cdot k|^{2}\right)+2|k|^{4}}{|k|^{3}\left(|k|^{2}+|k|-2 P \cdot k\right)} \\
& +\left\langle\psi_{n-1}\left|H_{f}\right| \psi_{n-1}\right\rangle \frac{1}{(2 \pi)^{2}} \int_{|k| \leq \Lambda} d^{3} k \frac{4\left(|P|^{2}|k|^{2}-|P \cdot k|^{2}\right)+2|k|^{4}}{|k|^{4}(1-2|P|)^{2}} .
\end{aligned}
$$

To estimate the term $I I_{n}$ we use the crude estimate

$$
\begin{array}{r}
\left|F\left(k_{n}\right) \psi_{n-1}\right| \leq\left(2\left|P \cdot G\left(k_{n}\right)\right|+3\left|H\left(k_{n}\right)\right|\right)\left|\psi_{n-1}\left(k_{1}, \ldots, k_{n-1}\right)\right| \\
\equiv\left|F\left(k_{n}\right)\right|\left|\psi_{n-1}\left(k_{1}, \ldots, k_{n-1}\right)\right|
\end{array}
$$


where the absolute values of $\psi_{n-1}, G$ and $H$ contain the appropriate norms in $\mathbb{C}^{2}$. On the $n$-photon space $\mathcal{Q} \geq(1-2|P|)\left(\left|k_{1}\right|+\left|k_{n}\right|+\alpha^{3 / 2}\right)$, so we get the upper bound

$$
\begin{aligned}
& I I_{n} \leq \frac{n-1}{1-2|P|} \int d^{3} k_{1} \cdots d^{3} k_{n} \\
& \quad \times \frac{\left|\psi_{n-1}\left(k_{1}, \ldots, k_{n-1}\right)\right|\left|F\left(k_{n}\right)\right|\left|F\left(k_{1}\right)\right|\left|\psi_{n-1}\left(k_{2}, \ldots, k_{n}\right)\right|}{\left|k_{1}\right|+\left|k_{n}\right|+\alpha^{3 / 2}} .
\end{aligned}
$$

Introducing the photon density

$$
\rho_{n-1}(k)=(n-1) \int d^{3} k_{2} \cdots d^{3} k_{n-1}\left|\psi_{n-1}\left(k, k_{2}, \ldots, k_{n-1}\right)\right|^{2}
$$

and using Schwarz' inequality twice, we estimate

$$
\begin{aligned}
& I I_{n} \leq \frac{1}{1-2|P|} \int d^{3} k_{1} d^{3} k_{n} \frac{\sqrt{\rho_{n-1}\left(k_{1}\right) \rho_{n-1}\left(k_{n}\right)}\left|F\left(k_{1}\right)\right|\left|F\left(k_{n}\right)\right|}{\left|k_{1}\right|+\left|k_{n}\right|+\alpha^{3 / 2}} \\
& \leq \frac{1}{1-2|P|}\left\langle\psi_{n-1}\left|H_{f}\right| \psi_{n-1}\right\rangle\left(\int d^{3} k_{1} d^{3} k_{n} \frac{\left|F\left(k_{1}\right)\right|^{2}\left|F\left(k_{n}\right)\right|^{2}}{\left|k_{1}\right|\left|k_{n}\right|\left(\left|k_{1}\right|+\left|k_{n}\right|+\alpha^{3 / 2}\right)^{2}}\right)^{\frac{1}{2}} .
\end{aligned}
$$

Since $|F(k)| \leq$ const. $|k|^{-1 / 2}$ for small $k$ the last term diverges logarithmically as $\alpha \rightarrow 0$.

Putting together (3.31) and (3.35) and using the a priori knowledge that $\left\langle\Psi_{0}\left|H_{f}\right| \Psi_{0}\right\rangle \leq$ const. $\alpha$ we get

$$
\begin{array}{r}
\sum_{n \geq 1}\left(I_{n}+I I_{n}\right) \leq \frac{1}{(2 \pi)^{2}} \int_{|k| \leq \Lambda} d^{3} k \frac{4\left(|P|^{2}|k|^{2}-|P \cdot k|^{2}\right)+2|k|^{4}}{|k|^{3}\left(|k|^{2}+|k|-2 P \cdot k\right)} \\
+O(\alpha \ln (1 / \alpha)) .
\end{array}
$$

We insert this into (3.20) and arrive at the lower bound

$$
\begin{array}{r}
\mathcal{E}_{P} \geq|P|^{2}+\frac{\alpha}{\pi} \Lambda^{2}-\frac{\alpha}{(2 \pi)^{2}} \int_{|k| \leq \Lambda} d^{3} k \frac{4\left(|P|^{2}|k|^{2}-|P \cdot k|^{2}\right)+2|k|^{4}}{|k|^{3}\left(|k|^{2}+|k|-2 P \cdot k\right)} \\
-O\left(\alpha^{3 / 2}\right) .
\end{array}
$$

Together with the upper bound (3.10) this proves (3.4), since

$$
\begin{aligned}
2|k|\left(\frac{1}{|k|^{2}+|k|-2 P \cdot k}-\frac{1}{|k|^{2}+|k|}\right) \\
=\frac{2|k|}{\left(|k|^{2}+|k|\right)^{2}}\left(2 P \cdot k+\frac{|2 P \cdot k|^{2}}{|k|^{2}+|k|-2 P \cdot k}\right),
\end{aligned}
$$

and the first term on the right side vanishes after integration over $k$. 
The coefficient of $|P|^{2}$ in an expansion of $\mathcal{E}_{P}$ around $P=0$ determines the physical mass $m$ as a function of $m_{0}, \alpha$ and $\Lambda$. Since

$$
\begin{array}{r}
\int_{|k| \leq \frac{\Lambda}{2 m_{0}}} d^{3} k \frac{|k|}{|k|^{2}+|k|}\left(\frac{|P|^{2}}{|k|^{2}}+|P \cdot k|^{2}\left(\frac{2}{\left(|k|^{2}+|k|\right)^{2}}-\frac{1}{|k|^{4}}\right)\right)= \\
|P|^{2} \frac{16 \pi}{3}\left[\ln \left(1+\frac{\Lambda}{2 m_{0}}\right)-\frac{3}{4} \frac{\Lambda\left(\Lambda+\frac{4}{3} m_{0}\right)}{\left(\Lambda+2 m_{0}\right)^{2}}\right]
\end{array}
$$

Equation (3.4) leads to the following relation of $m$ and $m_{0}$, to leading order in $\alpha$ :

$$
m=m_{0}\left(1+\alpha \frac{16}{3 \pi}\left[\ln \left(1+\frac{\Lambda}{2 m_{0}}\right)-\frac{3}{4} \frac{\Lambda\left(\Lambda+\frac{4}{3} m_{0}\right)}{\left(\Lambda+2 m_{0}\right)^{2}}\right]\right) .
$$

This relation is decisive when renormalizing the bare mass in the expression for the binding energy derived in the next section. Note that for any given positive values of $m, \alpha$ and $\Lambda$ there is a unique positive solution $m_{0}$ to the equation above. This is in contrast to the analogous expression in dipole approximation, where the term in square brackets is replaced by a factor linear in $\Lambda / m_{0}$, and consequently a positive solution only exists for $\alpha \Lambda$ small enough. More precisely, in dipole approximation the physical mass can be calculated explicitly, not only to leading order in $\alpha$, and the result is

$$
m=m_{0}+\frac{4 \alpha}{3 \pi} \Lambda .
$$

This was first shown by van Kampen in his thesis, as described in Kramers' biography [D, Sect. 16.III.D].

\section{Binding energy}

In this section we shall calculate the binding energy of an electron in the field of nucleus of charge $Z>0$. As we explained in the Introduction, we want to consider the case of small $\alpha$ with $Z \alpha$ not necessarily small. To avoid confusion, we denote the fine structure constant appearing in the external potential by $\beta$. I.e., we add to the Hamiltonian the potential

$$
V(x)=-\frac{\beta Z}{|x|}
$$

with $\beta \approx 1 / 137$. More general external potentials can be treated in the same manner, but for the sake of simplicity we shall not do so here. 
Let $e_{0}=-\frac{1}{2} m_{0}(\beta Z)^{2}$ be the ground state energy of the hydrogen atom without coupling to the photon field, and denote the corresponding ground state wave function by $\phi_{0}$. Let $\mathcal{E}(V)$ denote the ground state energy of (2.1). The existence of a ground state for $H$ has recently been shown in [GLL].

The following Theorem gives $\mathcal{E}(V)$ to leading order in $\alpha$. To obtain the binding energy, one has to subtract it from the self energy $\mathcal{E}(0)$, which will be done in Theorem 3. We introduce the notation that $A(x)=D(x)+D^{*}(x)$ and $B(x)=E(x)+E^{*}(x)$, where $D$ and $E$ denote the part of $A$ and $B$, respectively, containing only annihilation operators.

THEOREM 2 (Ground State Energy). With $\mathcal{A}$ denoting the operator $\mathcal{A}=\frac{1}{2 m_{0}}|p|^{2}+V-e_{0}+H_{f}$, the ground state energy of (2.1) with external potential (4.1) is given by

$$
\mathcal{E}(V)=e_{0}+\frac{\alpha}{2 m_{0} \pi} \Lambda^{2}-\frac{\alpha}{4 m_{0}^{2}}\left\langle\phi_{0}\left|4 p \cdot D \frac{1}{\mathcal{A}} p \cdot D^{*}+E \cdot \frac{1}{\mathcal{A}} E^{*}\right| \phi_{0}\right\rangle+O\left(\alpha^{3 / 2}\right) .
$$

Proof. We set again $m_{0}=\frac{1}{2}$ for simplicity, and start with the upper bound: We use as a trial state

$$
\Psi=\left\{\phi_{0} \uparrow, \frac{-\sqrt{\alpha}}{|p|^{2}+V-e_{0}+|k|}\left[2 p \cdot D^{*}+\sigma \cdot E^{*}\right] \phi_{0} \uparrow, 0, \ldots\right\},
$$

with $E^{*}, D^{*}$ and $\phi_{0}$ as explained above. Note that in contrast to (3.5) there is no need for an infrared cut-off, since the norm of $\Psi$ is finite. This can be seen as follows. An infrared problem can only arise from the overlap of $p \cdot D^{*} \phi_{0}$ with $\phi_{0}$, since $|p|^{2}+V-e_{0}$ has a spectral gap above zero. Since $p \phi_{0}$ is orthogonal to $\phi_{0}$, we can estimate the projection of $p \cdot D^{*} \phi_{0}$ onto $\phi_{0}$ (for fixed $k$ ) by

$$
\begin{aligned}
& \left|\left\langle\phi_{0} \mid p \cdot D^{*} \phi_{0}\right\rangle\right|=\left|G(k) \cdot\left\langle\phi_{0} \mid e^{-i k \cdot x} p \phi_{0}\right\rangle\right| \\
& =\left|G(k) \cdot\left\langle\phi_{0} \mid\left(e^{-i k \cdot x}-1\right) p \phi_{0}\right\rangle\right| \leq|G(k)||k|\left\|p \phi_{0}\right\|^{2}\left\|x \phi_{0}\right\|^{2},
\end{aligned}
$$

where we used that $\left|e^{-i k \cdot x}-1\right| \leq|k||x|$. The thus obtained additional factor $|k|$ makes the integral over $k$ finite.

Similarly to the calculation in the previous section, we obtain

$$
\begin{aligned}
& \langle\Psi|H| \Psi\rangle=\left(e_{0}+\frac{\alpha}{\pi} \Lambda^{2}\right)\langle\Psi \mid \Psi\rangle \\
& -\alpha\left\langle\left(2 p \cdot D^{*}+\sigma \cdot E^{*}\right) \phi_{0} \uparrow\left|\frac{1}{\mathcal{A}}\right|\left(2 p \cdot D^{*}+\sigma \cdot E^{*}\right) \phi_{0} \uparrow\right\rangle+O\left(\alpha^{2}\right) .
\end{aligned}
$$


Using the anti-commutation relations for $\sigma$, we see that the mixed terms vanish, and we arrive at (4.2) as an upper bound. More precisely,

$$
\Re\left\langle p \cdot D^{*} \phi_{0} \uparrow\left|\frac{1}{\mathcal{A}}\right| \sigma \cdot E^{*} \phi_{0} \uparrow\right\rangle=0
$$

and

$$
\Im\left\langle E_{i}^{*} \phi_{0}\left|\frac{1}{\mathcal{A}}\right| E_{j}^{*} \phi_{0}\right\rangle=0,
$$

as can be seen by using the fact the $G$ is real and $H$ is purely imaginary, and that the operator $\mathcal{A}$ commutes with the reflection $x \rightarrow-x$.

Lower bound: Proceeding as in the proof of the lower bound in Theorem 1 (c.f. also [H]), it is easy to see that, in the ground state $\Psi_{0},\left\langle\Psi_{0}\left|H_{f}\right| \Psi_{0}\right\rangle \leq$ const. $\alpha$. Moreover,

$$
\begin{aligned}
\left\langle\Psi_{0}|H| \Psi_{0}\right\rangle \geq & e_{0}+\frac{\alpha}{\pi} \Lambda^{2}-\text { const. } \alpha^{3 / 2} \\
& +\left\langle\left.\Psi_{0}|| p\right|^{2}+V-e_{0}+2 \sqrt{\alpha} p \cdot A+\sqrt{\alpha} \sigma \cdot B+H_{f} \mid \Psi_{0}\right\rangle
\end{aligned}
$$

(compare with (3.20)). The expression in the second line can be rewritten as

$$
\begin{aligned}
\left\langle\left.\psi_{0}|| p\right|^{2}+V-e_{0} \mid \psi_{0}\right\rangle+\sum_{n \geq 1}\langle & \left.\psi_{n}|| p\right|^{2}+V-e_{0}+H_{f}\left|\psi_{n}\right\rangle \\
& +2 \sqrt{\alpha} \Re\left\langle\psi_{n}\left|2 p \cdot D^{*}+\sigma \cdot E^{*}\right| \psi_{n-1}\right\rangle .
\end{aligned}
$$

With $\mathcal{A}$ as stated in the theorem and $F^{*}=2 p \cdot D^{*}+\sigma \cdot E^{*}$, we get as a lower bound for (4.9), using Schwarz' inequality,

$$
\left\langle\psi_{0}|\mathcal{A}| \psi_{0}\right\rangle-\alpha \sum_{n \geq 0}\left\langle\psi_{n}\left|F \cdot \frac{1}{\mathcal{A}} F^{*}\right| \psi_{n}\right\rangle
$$

To bound the terms with $n \geq 1$ from below, we need the following Lemma.

LEMMA 1. Let $\mathcal{N}=d \Gamma(1)$ denote the number operator. Then

$$
F \cdot \frac{1}{\mathcal{A}} F^{*} \leq \text { const. }(1+\mathcal{N}) \text {. }
$$

Proof. By Schwarz' inequality

$$
F \cdot \frac{1}{\mathcal{A}} F^{*} \leq 2 \sigma \cdot E \frac{1}{\mathcal{A}} \sigma \cdot E^{*}+8 D \cdot p \frac{1}{\mathcal{A}} p \cdot D^{*} .
$$


We first consider the last term. Let $p_{i}, i=1,2,3$, denote the components of $p$. We claim that

$$
p_{i} \frac{1}{|p|^{2}+V-e_{0}+H_{f}} p_{i} \leq 1+\frac{\left|e_{0}\right|}{H_{f}} .
$$

It suffices to prove this for $H_{f}$ some positive number $\mu$. Since

$$
\varepsilon p_{i}^{2} \leq \varepsilon|p|^{2} \leq|p|^{2}+V-e_{0} /(1-\varepsilon)
$$

for $0 \leq \varepsilon<1$, we get

$$
p_{i} \frac{1}{|\dot{p}|^{2}+V-e_{0} /(1-\varepsilon)} p_{i} \leq \frac{1}{\varepsilon} .
$$

With $\varepsilon=\mu /\left(\mu+\left|e_{0}\right|\right)$ this gives

$$
p_{i} \frac{1}{|p|^{2}+V-e_{0}+\mu} p_{i} \leq 1+\frac{\left|e_{0}\right|}{\mu} .
$$

Using that $\mathcal{A} \geq H_{f}$ and again Schwarz' inequality for the terms with $p_{i}$ and $p_{j}$ for $i \neq j$, we thus obtain

$$
F \cdot \frac{1}{\mathcal{A}} F^{*} \leq 2 \sigma \cdot E \frac{1}{H_{f}} \sigma \cdot E^{*}+24 D \cdot\left(1+\frac{\left|e_{0}\right|}{H_{f}}\right) D^{*} .
$$

Consider now the operator $D_{i}\left(1+\frac{\left|e_{0}\right|}{H_{f}}\right) D_{i}^{*}$ restricted to the $(n-1)$-photon sector. By use of Schwarz' inequality we can estimate

$$
\begin{aligned}
\left\langle\psi_{n}\right| D_{i}(1+ & \left.\frac{\left|e_{0}\right|}{H_{f}}\right) D_{i}^{*}\left|\psi_{n}\right\rangle \\
& \leq n\left\langle\psi_{n-1} e^{i k_{n} \cdot x} G_{i}\left(k_{n}\right)\left|1+\frac{\left|e_{0}\right|}{H_{f}}\right| \psi_{n-1} e^{i k_{n} \cdot x} G_{i}\left(k_{n}\right)\right\rangle
\end{aligned}
$$

where $\psi_{n-1}$ is short for $\psi_{n-1}\left(x, k_{1}, \ldots, k_{n-1}\right)$. Since $H_{f} \geq\left|k_{n}\right|$ in the $n$-photon sector, the last expression is bounded above by

$$
n\left\|\psi_{n}\right\|^{2} \sum_{\lambda=1}^{2} \int d^{3} k\left|G_{i}^{\lambda}(k)\right|^{2}\left(1+\frac{\left|e_{0}\right|}{|k|}\right) .
$$

Proceeding analogously for the other terms in (4.17) we prove the desired result.

From [GLL, Thm. 6.1] we infer that in the ground state $\Psi_{0}$ we have $\left\langle\Psi_{0}|\mathcal{N}| \Psi_{0}\right\rangle \leq$ const. $\alpha$, where the constant depends only on $\Lambda$. Using this we conclude from the Lemma above that

$$
(4.10) \geq\left\langle\psi_{0}|\mathcal{A}| \psi_{0}\right\rangle-\alpha\left\langle\psi_{0}\left|F \cdot \frac{1}{\mathcal{A}} F^{*}\right| \psi_{0}\right\rangle-\text { const. } \alpha^{2},
$$


and also that $\left\|\psi_{0}\right\|^{2} \geq 1$ - const. $\alpha$. It therefore follows from first order perturbation theory $[\mathrm{K}]$ that, for small enough $\alpha$,

$$
(4.20) \geq-\alpha\left\langle\phi_{0} \uparrow\left|F \cdot \frac{1}{\mathcal{A}} F^{*}\right| \phi_{0} \uparrow\right\rangle-\text { const. } \alpha^{2} .
$$

The spin $\uparrow$ is just one possible choice, any other spin will do. As already noted in the upper bound (c.f. (4.6) and (4.7)),

$$
\left\langle\phi_{0} \uparrow\left|F \cdot \frac{1}{\mathcal{A}} F^{*}\right| \phi_{0} \uparrow\right\rangle=\left\langle\phi_{0}\left|4 p \cdot D \frac{1}{\mathcal{A}} p \cdot D^{*}+E \cdot \frac{1}{\mathcal{A}} E^{*}\right| \phi_{0}\right\rangle,
$$

which finishes the proof of the lower bound.

With the expression for the ground state energy $\mathcal{E}(V)$ in hand, we can subtract it from the self energy to obtain the binding energy. This generalizes a result in $[\mathrm{H}]$, where a lower bound on the binding energy was obtained. Moreover, the self energy was calculated, to leading order in $\alpha$, in $[\mathrm{H}]$, and we will use the result from there. Note that both the ground state energy and the self energy are, to order $\alpha$, linearly divergent in $\Lambda$ (for fixed $m_{0}$ ). The binding energy only shown a logarithmic divergence, which will be removed by mass renormalization in the next section.

THEOREM 3 (Binding Energy). With $\mathcal{B}$ denoting the operator $\mathcal{B}=$ $\frac{1}{2 m_{0}}|p-k|^{2}+V-e_{0}+|k|$, the binding energy of (2.1) with external potential (4.1) is given by

$$
\begin{aligned}
& \mathcal{E}(0)-\mathcal{E}(V)=-e_{0}+\frac{\alpha}{m_{0}^{2}} \sum_{i, j=1}^{3}\left\langle p_{i} \phi_{0} \otimes G_{i}\left|\frac{1}{\mathcal{B}}\right| p_{j} \phi_{0} \otimes G_{j}\right\rangle \\
& +\frac{\alpha}{m_{0}^{2}} \sum_{i=1}^{3}\left\langle\phi_{0} \otimes H_{i}\left|\frac{k \cdot p}{|k|^{2}+2 m_{0}|k|} \frac{1}{\mathcal{B}} \frac{k \cdot p}{|k|^{2}+2 m_{0}|k|}\right| \phi_{0} \otimes H_{i}\right\rangle+O\left(\alpha^{3 / 2}\right) .
\end{aligned}
$$

Proof. We set again $m_{0}=\frac{1}{2}$ for simplicity. From [H, Thm. 1] we know that

$$
\begin{aligned}
\mathcal{E}(0) & =\frac{\alpha}{\pi} \Lambda^{2}-\alpha\left\langle 0\left|E(0) \cdot \frac{1}{|k|^{2}+|k|} E^{*}(0)\right| 0\right\rangle+O\left(\alpha^{2}\right) \\
& =\frac{2 \alpha}{\pi}(\Lambda-\ln (1+\Lambda))+O\left(\alpha^{2}\right)
\end{aligned}
$$

where $|0\rangle$ denotes the vacuum in $\mathcal{F}_{b}$. We claim that the vacuum expectation value in the first line of (4.24) can be written as

$$
-\alpha\left\langle\phi_{0}\left|E \cdot \frac{1}{|p+k|^{2}+V-e_{0}+|k|^{2}+|k|} E^{*}\right| \phi_{0}\right\rangle,
$$


where $E=E(x)$. To see this, consider the unitary transformation $U$ on the one-photon space $L^{2}\left(\mathbb{R}^{3}, d^{3} x ; \mathbb{C}^{2}\right) \otimes L^{2}\left(\mathbb{R}^{3}, d^{3} k ; \mathbb{C}^{2}\right)$ given by

$$
(U \psi)(x, k)=e^{i k \cdot x} \psi(x, k)
$$

suppressing the dependence on spin and polarization in the notation. We have

$$
\left(U E_{i}^{*} \phi_{0}\right)(x, k)=H_{i}(k) \phi_{0}(x)=\phi_{0} \otimes H_{i}
$$

Moreover,

$$
\begin{aligned}
U\left(|p+k|^{2}+V(x)-e_{0}\right. & \left.+|k|^{2}+|k|\right) U^{*}=|p|^{2}+V(x)-e_{0}+|k|^{2}+|k| \\
& =\left(|p|^{2}+V-e_{0}\right) \otimes 1+1 \otimes\left(|k|^{2}+|k|\right), \quad
\end{aligned}
$$

which proves the claim.

Introducing the notation $\mathcal{B}^{\prime}=|p|^{2}+V-e_{0}+|k|^{2}+|k|=\mathcal{B}+2 p \cdot k$ and using this unitary transformation, we have

$$
\begin{aligned}
\left\langle\phi_{0}\left|E \cdot \frac{1}{\mathcal{B}} E^{*}\right| \phi_{0}\right\rangle- & \left\langle\phi_{0}\left|E \cdot \frac{1}{|p+k|^{2}+V-e_{0}+|k|^{2}+|k|} E^{*}\right| \phi_{0}\right\rangle \\
& =\sum_{i=1}^{3}\left\langle\phi_{0} \otimes H_{i}\left|\frac{1}{\mathcal{B}}-\frac{1}{\mathcal{B}^{\prime}}\right| \phi_{0} \otimes H_{i}\right\rangle \\
= & \sum_{i=1}^{3}\left\langle\phi_{0} \otimes H_{i}\left|\frac{1}{\mathcal{B}^{\prime}} 2 p \cdot k \frac{1}{\mathcal{B}^{\prime}}+\frac{1}{\mathcal{B}^{\prime}} 2 p \cdot k \frac{1}{\mathcal{B}} 2 p \cdot k \frac{1}{\mathcal{B}^{\prime}}\right| \phi_{0} \otimes H_{i}\right\rangle .
\end{aligned}
$$

The first term in the last expression is zero by symmetry with respect to the reflection $x \rightarrow-x$. Moreover, $\mathcal{B}^{\prime-1} \phi_{0} \otimes H_{i}=\phi_{0} \otimes H_{i} /\left(|k|^{2}+|k|\right)$. Hence, using Theorem 2 and (4.24), and the unitary transformation (4.26), we obtain, up to an error not bigger that order $\alpha^{3 / 2}$,

$$
\begin{aligned}
\mathcal{E}(0)-\mathcal{E}(V)= & -e_{0}+4 \alpha \sum_{i, j=1}^{3}\left\langle p_{i} \phi_{0} \otimes G_{i}\left|\frac{1}{\mathcal{B}}\right| p_{j} \phi_{0} \otimes G_{j}\right\rangle \\
& +\alpha \sum_{i=1}^{3}\left\langle\phi_{0} \otimes H_{i}\left|\frac{2 k \cdot p}{|k|^{2}+|k|} \frac{1}{\mathcal{B}} \frac{2 k \cdot p}{|k|^{2}+|k|}\right| \phi_{0} \otimes H_{i}\right\rangle,
\end{aligned}
$$

proving the Theorem. 


\section{Renormalization}

In the previous section, we calculated the binding energy to leading order in $\alpha$. However, the bare mass enters this calculation, which is not a physical quantity. We have to replace it by the physical mass, which was, to leading order in $\alpha$, calculated in Section 3. Note that both expressions show a logarithmic dependence on $\Lambda$. It turns out the these divergences cancel and a finite result will be obtained.

In the two terms in (4.23) of order $\alpha$ we can simply replace $m_{0}$ by $m$ since we are only interested in a leading order calculation. This also affects $e_{0}$ and $\phi_{0}$; i.e., from now on $e_{0}=-\frac{1}{2} m(\beta Z)^{2}$ and $\phi_{0}$ is the ground state wave function of $\frac{1}{2 m}|p|^{2}+V$.

The $m_{0}$ in the first term $e_{0}$ in (4.23) has to be replaced by the expression (3.40), however. This leads to

$$
\begin{array}{r}
\mathcal{E}(0)-\mathcal{E}(V)=\frac{m}{2}(\beta Z)^{2}\left(1-\alpha \frac{16}{3 \pi}\left[\ln \left(1+\frac{\Lambda}{2 m}\right)-\frac{3}{4} \frac{\Lambda\left(\Lambda+\frac{4}{3} m\right)}{(\Lambda+2 m)^{2}}\right]\right) \\
+\frac{\alpha}{m^{2}} \sum_{i, j=1}^{3}\left\langle p_{i} \phi_{0} \otimes G_{i}\left|\frac{1}{\mathcal{B}}\right| p_{j} \phi_{0} \otimes G_{j}\right\rangle \\
+\frac{\alpha}{m^{2}} \sum_{i=1}^{3}\left\langle\phi_{0} \otimes H_{i}\left|\frac{k \cdot p}{|k|^{2}+2 m|k|} \frac{1}{\mathcal{B}} \frac{k \cdot p}{|k|^{2}+2 m|k|}\right| \phi_{0} \otimes H_{i}\right\rangle
\end{array}
$$

with $\mathcal{B}=\frac{1}{2 m}|p-k|^{2}+V-e_{0}+|k|$. We suppress higher order terms in $\alpha$ from now on.

We shall show that (5.1) has a finite limit as $\Lambda \rightarrow \infty$, i.e., the cut-off can be removed. Let $\mathcal{B}^{\prime}$ denote the operator $\mathcal{B}^{\prime}=\frac{1}{2 m}|p|^{2}+V-e_{0}+\frac{1}{2 m}|k|^{2}+|k|=$ $\mathcal{B}+p \cdot k / m$. An easy calculation, using (2.8) and (2.9) and the fact that

$$
\sum_{\lambda} G_{i}^{\lambda}(k) G_{j}^{\lambda}(k)=\frac{1}{(2 \pi)^{2}|k|^{3}}\left(|k|^{2} \delta_{i j}-k_{i} k_{j}\right)
$$


gives

$$
\begin{aligned}
& \sum_{i, j=1}^{3}\left\langle p_{i} \phi_{0} \otimes G_{i}\left|\frac{1}{\mathcal{B}^{\prime}}\right| p_{j} \phi_{0} \otimes G_{j}\right\rangle \\
& +\sum_{i=1}^{3}\left\langle\phi_{0} \otimes H_{i}\left|\frac{k \cdot p}{|k|^{2}+2 m|k|} \frac{1}{\mathcal{B}^{\prime}} \frac{k \cdot p}{|k|^{2}+2 m|k|}\right| \phi_{0} \otimes H_{i}\right\rangle \\
& \quad=m \sum_{j=1}^{3}\left\langle p_{j} \phi_{0}\left|f\left(\frac{\frac{1}{2 m}|p|^{2}+V-e_{0}}{2 m}, \frac{\Lambda}{2 m}\right)\right| p_{j} \phi_{0}\right\rangle
\end{aligned}
$$

Here $f$ is the function

$$
f(e, \Lambda)=\frac{4}{3 \pi} \int_{0}^{\Lambda} d k \frac{k^{5}}{e+k^{2}+k}\left(\frac{1}{k^{4}}+\frac{1}{\left(k^{2}+k\right)^{2}}\right) .
$$

Note that $\sum_{j}\left\langle p_{j} \phi_{0} \mid p_{j} \phi_{0}\right\rangle=2 m\left|e_{0}\right|$, and that

$$
f(0, \Lambda)=\frac{8}{3 \pi}\left(\ln (1+\Lambda)-\frac{3}{4} \frac{\Lambda\left(\Lambda+\frac{2}{3}\right)}{(\Lambda+1)^{2}}\right) .
$$

Therefore (5.1) can be written as

$$
\begin{aligned}
\mathcal{E}(0)-\mathcal{E}(V) & =\frac{m}{2}(\beta Z)^{2} \\
+ & \frac{\alpha}{m} \sum_{j=1}^{3}\left\langle p_{j} \phi_{0}\left|f\left(\frac{\frac{1}{2 m}|p|^{2}+V-e_{0}}{2 m}, \frac{\Lambda}{2 m}\right)-f\left(0, \frac{\Lambda}{2 m}\right)\right| p_{j} \phi_{0}\right\rangle \\
& \quad+\frac{\alpha}{m^{2}} \sum_{i, j=1}^{3}\left\langle p_{i} \phi_{0} \otimes G_{i}\left|\left(\frac{1}{\mathcal{B}}-\frac{1}{\mathcal{B}^{\prime}}\right)\right| p_{j} \phi_{0} \otimes G_{j}\right\rangle \\
+ & \frac{\alpha}{m^{2}} \sum_{i=1}^{3}\left\langle\phi_{0} \otimes H_{i}\left|\frac{k \cdot p}{|k|^{2}+2 m|k|}\left(\frac{1}{\mathcal{B}}-\frac{1}{\mathcal{B}^{\prime}}\right) \frac{k \cdot p}{|k|^{2}+2 m|k|}\right| \phi_{0} \otimes H_{i}\right\rangle .
\end{aligned}
$$

By definition $\mathcal{B}=\mathcal{B}^{\prime}-b$ with $b=p \cdot k / m$. However, it is easy to see that the expressions (5.1) and (5.6) do not change if we replace $\mathcal{B}$ by $\mathcal{B}^{\prime}+b$. Therefore we can replace $1 / \mathcal{B}$ by $\frac{1}{2}\left(1 /\left(\mathcal{B}^{\prime}-b\right)+1 /\left(\mathcal{B}^{\prime}+b\right)\right)$, and instead of $1 / \mathcal{B}-1 / \mathcal{B}^{\prime}$ in $(5.6)$ we can write

$$
\frac{1}{2}\left(\frac{1}{\mathcal{B}^{\prime}-b}+\frac{1}{\mathcal{B}^{\prime}+b}\right)-\frac{1}{\mathcal{B}^{\prime}}=\frac{1}{\mathcal{B}^{\prime}} b \frac{1}{\mathcal{B}^{\prime}-b \mathcal{B}^{\prime-1} b} b \frac{1}{\mathcal{B}^{\prime}} .
$$


For given operators $h$ and $p$ let $C_{k}(h, p)$ denote the operator

$$
\begin{aligned}
& C_{k}(h, p)=\frac{1}{h+|k|^{2}+|k|} p \cdot k \times \\
& \quad \times \frac{1}{h+|k|^{2}+|k|-p \cdot k\left(h+|k|^{2}+|k|\right)^{-1} p \cdot k} p \cdot k \frac{1}{h+|k|^{2}+|k|} .
\end{aligned}
$$

We define the operator valued matrix $\mathcal{T}^{\Lambda}$ by its components

$$
\begin{aligned}
& \mathcal{T}_{i j}^{\Lambda}(h, p)= \\
& \quad \frac{1}{2 \pi^{2}} \int_{|k| \leq \Lambda} d^{3} k\left(\frac{2|k| k_{i} k_{j}}{\left(|k|^{2}+|k|\right)^{2}}+\frac{1}{|k|^{3}}\left(|k|^{2} \delta_{i j}-k_{i} k_{j}\right)\right) C_{k}(h, p),
\end{aligned}
$$

being an operator on $L^{2}\left(\mathbb{R}^{3}, d^{3} x ; \mathbb{C}^{2}\right) \otimes \mathbb{C}^{3}$. Using again (2.8), (2.9) and the orthogonality relations of $\varepsilon_{\lambda}(k)$ and $k$, we can rewrite (5.6) as

$$
\begin{aligned}
\mathcal{E}(0)- & \mathcal{E}(V)=\frac{m}{2}(\beta Z)^{2} \\
+ & \frac{\alpha}{m} \sum_{j=1}^{3}\left\langle p_{j} \phi_{0}\left|f\left(\frac{\frac{1}{2 m}|p|^{2}+V-e_{0}}{2 m}, \frac{\Lambda}{2 m}\right)-f\left(0, \frac{\Lambda}{2 m}\right)\right| p_{j} \phi_{0}\right\rangle \\
& +\frac{\alpha}{m} \sum_{i, j=1}^{3}\left\langle p_{i} \phi_{0}\left|\mathcal{T}_{i j}^{\Lambda /(2 m)}\left(\frac{\frac{1}{2 m}|p|^{2}+V-e_{0}}{2 m}, \frac{p}{m}\right)\right| p_{j} \phi_{0}\right\rangle .
\end{aligned}
$$

Both terms on the right side have a nice limit as $\Lambda \rightarrow \infty$. We define

$$
\begin{aligned}
\mathcal{S}(e)=\lim _{\Lambda \rightarrow \infty} & f(0, \Lambda)-f(e, \Lambda) \\
= & \frac{4}{3 \pi} \int_{0}^{\infty} d k \frac{e k^{5}}{\left(k^{2}+k\right)\left(e+k^{2}+k\right)}\left(\frac{1}{k^{4}}+\frac{1}{\left(k^{2}+k\right)^{2}}\right)
\end{aligned}
$$

and $\mathcal{T}(h, p)$ by

$$
\mathcal{T}_{i j}(h, p)=\mathcal{T}_{i j}^{\infty}(h, p) .
$$

Note that this operator, for $h=\frac{1}{2 m}|p|^{2}+V-e_{0}$, is well defined. To see this, we estimate

$$
\frac{1}{\mathcal{B}^{\prime}-b \mathcal{B}^{\prime-1} b} \leq \frac{1}{|k|}
$$

and

$$
\frac{1}{m^{2}}(p \cdot k)^{2} \leq \frac{1}{m^{2}}|p|^{2}|k|^{2} \leq|k|^{2} \frac{2}{\varepsilon m}\left(\frac{1}{2 m}|p|^{2}+V-e_{0} /(1-\varepsilon)\right)
$$


for all $0<\varepsilon<1$. Since $\mathcal{B}^{\prime} \geq|k|^{2} /(2 m)+|k|$ we get

$$
\mathcal{T}\left(\frac{\frac{1}{2 m}|p|^{2}+V-e_{0}}{2 m}, \frac{p}{m}\right) \leq \frac{2}{\varepsilon m} I_{\mathbb{C}^{3}}\left(\frac{1}{2 m}|p|^{2}+V-e_{0} /(1-\varepsilon)\right) \frac{16}{9 \pi}
$$

as an operator on $L^{2}\left(\mathbb{R}^{3}, d x ; \mathbb{C}^{2}\right) \otimes \mathbb{C}^{3}$. The last factor comes from the integral

$$
\frac{1}{3 \pi^{2}} \int_{\mathbb{R}^{3}} d^{3} k \frac{|k|^{4}}{\left(|k|^{2}+|k|\right)^{2}}\left(\frac{1}{|k|^{4}}+\frac{1}{\left(|k|^{2}+|k|\right)^{2}}\right)=\frac{16}{9 \pi} .
$$

We see that, for $h=\frac{1}{2 m}|p|^{2}+V-e_{0}$, both the operators $\mathcal{T}^{\Lambda}(h / 2 m, p / m)$ and $f(0, \Lambda)-f(h / 2 m, \Lambda)$ are monotone increasing in $\Lambda$, and bounded by const. $(1+h)$ independent of $\Lambda$. Passing to the limit $\Lambda \rightarrow \infty$, we have thus proved:

THEOREM 4 (Renormalized Binding Energy). To leading order in $\alpha$, the renormalized binding energy, after removing the cut-off, is given by

$$
\begin{aligned}
\mathcal{E}(0)-\mathcal{E}(V) & =\frac{m}{2}(\beta Z)^{2}-\frac{\alpha}{m} \sum_{j=1}^{3}\left\langle p_{j} \phi_{0}\left|\mathcal{S}\left(\frac{\frac{1}{2 m}|p|^{2}+V-e_{0}}{2 m}\right)\right| p_{j} \phi_{0}\right\rangle \\
& +\frac{\alpha}{m} \sum_{i, j=1}^{3}\left\langle p_{i} \phi_{0}\left|\mathcal{T}_{i j}\left(\frac{\frac{1}{2 m}|p|^{2}+V-e_{0}}{2 m}, \frac{p}{m}\right)\right| p_{j} \phi_{0}\right\rangle,
\end{aligned}
$$

with $\mathcal{S}$ and $\mathcal{T}$ the positive operators defined in (5.11), (5.12) and (5.9).

Note that, by scaling, the right side of (5.17) is $m$ times a function of $\beta Z$. This is of course clear from the viewpoint of physical dimensions, since, after removing $\Lambda, m$ is the only energy scale in the problem.

The function $\mathcal{S}$ is monotone increasing and concave, with

$$
\mathcal{S}(e) \approx \frac{4}{3 \pi} e \ln (1 / e) \quad \text { for } e \ll 1,
$$

and

$$
\mathcal{S}(e) \approx \frac{4}{3 \pi} \ln (e) \text { for } e \gg 1 .
$$

Because of the logarithmic factor appearing in (5.18), the right side of (5.15) is, for $e_{0} \ll m$, much smaller than the second term in (5.17). I.e., for $Z \ll 1 / \beta \approx 137$, the term with $\mathcal{T}$ is negligible compared to the term with $\mathcal{S}$.

Note that if we neglect $\mathcal{T}$ and approximate $\mathcal{S}(e)$ by $\frac{4}{3 \pi} e \ln (1 / e)$ we obtain exactly Bethe's result for the ground state energy shift [Be]. For this 
result it is not necessary to include the $B$ field in the Hamiltonian, since the logarithmic factor in $\mathcal{S}(e)$ for small $e$ is entirely due to the term $1 /|k|^{4}$ in (5.11), which comes from the $p \cdot A$ term in the Hamiltonian. The part with $1 /\left(|k|^{2}+|k|\right)^{2}$, which stems from the $\sigma \cdot B$ term, is, when divided by $e$, bounded as $e \rightarrow 0$.

For $\beta Z$ not too small, there is a significant difference between our expression (5.17) and Bethe's formula. First of all, there is a different behavior of $\mathcal{S}(e)$ for large $e$, and secondly the term $\mathcal{T}$ contributes, with a different sign than $\mathcal{S}$. It would be interesting to evaluate the terms in (5.17) numerically, given the physical values of $\alpha=\beta$ and $Z$. For small $Z$, the result will essentially agree with Bethe's expression, whereas for larger values of $Z$ our formula should be closer to the experimentally observed value.

Remark. The appearance of the second term $\mathcal{T}$ in Theorem 4 is essentially due to the fact that the physical mass was obtained at total momentum $P=0$, whereas the ground state of an electron in the field of a nucleus shows a definite momentum distribution. In fact, a definition of the physical mass of the state $\phi_{0}$ by the relation

$$
\left\langle\phi_{0}\left|\mathcal{E}_{p}-\mathcal{E}_{0}\right| \phi_{0}\right\rangle=\frac{1}{2 m}\left\langle\left.\phi_{0}|| p\right|^{2} \mid \phi_{0}\right\rangle
$$

where $p=-i \nabla_{x}$ is the electron momentum operator, would account for a subtraction of a term similar to $\mathcal{T}$. From a physical point of view, however, this definition is not very satisfactory since one wants to compare the binding energy with the rest mass of the electron and not with a mass defined in dependence on the state of the system.

To see the order of magnitude of the shift of the binding energy due to the presence of the quantized radiation field to leading order in $\alpha$, we now present a rough lower bound. The last term in (5.17) is positive and can be neglected. For the first term, we use concavity of $\mathcal{S}$ and Jensen's inequality to get

$$
\begin{aligned}
& \mathcal{E}(0)-\mathcal{E}(V) \geq \frac{m}{2}(\beta Z)^{2} \\
& -\frac{\alpha}{m} \sum_{j=1}^{3}\left\langle p_{j} \phi_{0} \mid p_{j} \phi_{0}\right\rangle \mathcal{S}\left(\frac{1}{2 m} \frac{\sum_{j=1}^{3}\left\langle\left. p_{j} \phi_{0}\left|\frac{1}{2 m}\right| p\right|^{2}+V-e_{0} \mid p_{j} \phi_{0}\right\rangle}{\sum_{j=1}^{3}\left\langle p_{j} \phi_{0} \mid p_{j} \phi_{0}\right\rangle}\right) .
\end{aligned}
$$


Using that $\sum_{j=1}^{3}\left\langle p_{j} \phi_{0} \mid p_{j} \phi_{0}\right\rangle=2 m\left|e_{0}\right|$ and

$$
\begin{aligned}
& \sum_{j=1}^{3}\left\langle\left. p_{j} \phi_{0}\left|\frac{1}{2 m}\right| p\right|^{2}+V-e_{0} \mid p_{j} \phi_{0}\right\rangle \\
&=-\frac{1}{2} \sum_{j=1}^{3}\left\langle\phi_{0}\left|\left[p_{j},\left[p_{j}, \frac{1}{2 m}|p|^{2}+V-e_{0}\right]\right]\right| \phi_{0}\right\rangle \\
&=2 \pi(\beta Z)\left|\phi_{0}(0)\right|^{2}=2 m^{3}(\beta Z)^{4}
\end{aligned}
$$

we get

$$
\mathcal{E}(0)-\mathcal{E}(V) \geq \frac{m}{2}(\beta Z)^{2}-m \alpha(\beta Z)^{2} \mathcal{S}\left((\beta Z)^{2}\right) .
$$

In nature $\alpha=\beta$, and therefore

$$
\mathcal{E}(0)-\mathcal{E}(V) \geq \frac{m}{2}(\beta Z)^{2}\left[1-2 \beta \mathcal{S}\left((\beta Z)^{2}\right)\right] .
$$

Inserting $\beta=1 / 137$ and $Z=1$ for the hydrogen atom gives a lower bound on the shift of the binding energy of $-12.09 * 10^{3} \mathrm{MHz}$, which is off the experimental value by a factor of about 1.5 (see, e.g., the appendix of [Ki] for a detailed discussion). This is of course only a rough lower bound, the true value given by (5.17) is probably much closer. It should be noted, however, that the shift considered here is only the one due to the quantized radiation field, which is smaller than the shift due to relativistic effects.

\section{Lamb Shift}

Due to the presence of the quantized photon field, the ground state is the only eigenstate of the Hamiltonian [BFS]. All the excited states that exist without radiation field turn into resonances. These are metastable states that decay after a characteristic lifetime.

A reasonable guess for the energies of these resonances to leading order in the coupling $\alpha$ is to compute the expectation value of the Hamiltonian in a state similar to the one we used for the ground state and that has proved to give the right answer to leading order. I.e., we consider a state containing only one photon, but replace $\phi_{0}$ and $e_{0}$ by some excited state of the unperturbed hydrogen atom with corresponding energy. We denote by $\phi_{n, l}$ an eigenfunction of $\frac{1}{2 m}|p|^{2}+V$ with principal quantum number $n$, angular momentum $l$, and corresponding energy $e_{n}$ (which is, of course, independent of $l$ ). Doing this calculation and also the mass renormalization to leading 
order in $\alpha$, it is therefore natural to expect an energy of the metastable excited states, to leading order in $\alpha$, as

$$
\begin{aligned}
-\frac{m(\beta Z)^{2}}{2 n^{2}} & +\frac{\alpha}{m} \sum_{j=1}^{3}\left\langle p_{j} \phi_{n, l}\left|\mathcal{S}\left(\frac{\frac{1}{2 m}|p|^{2}+V-e_{n}}{2 m}\right)\right| p_{j} \phi_{n, l}\right\rangle \\
& -\frac{\alpha}{m} \sum_{i, j=1}^{3}\left\langle p_{i} \phi_{n, l}\left|\mathcal{T}_{i j}\left(\frac{\frac{1}{2 m}|p|^{2}+V-e_{n}}{2 m}, \frac{p}{m}\right)\right| p_{j} \phi_{n, l}\right\rangle
\end{aligned}
$$

Note that the operator $\frac{1}{2 m}|p|^{2}+V-e_{n}+\frac{1}{2 m}|k|^{2}+|k|$ is not positive, but nevertheless invertible for almost every $k$. The expression (6.1) makes perfect sense if the integrals over $k$ in (5.11) and (5.9) are interpreted as the Cauchy principal value.

The same discussion as for the ground state energy shift in the previous section also applies here. Neglecting $\mathcal{T}$ and approximating $\mathcal{S}(e)$ by $\frac{4}{3 \pi} e \ln (1 / e)$ gives the result of Bethe [Be]. This approximation is valid for $Z \ll 1 / \alpha$. For larger $Z$, our formula presumably provides a better description of the energy level shift due to the presence of the quantized radiation field than Bethe's formula.

The correctness of the formula for the shift of the energy levels can best be tested on the classical Lamb shift, namely the splitting of the $2 s_{1 / 2}$ and $2 p_{1 / 2}$ states, since this is an effect entirely due to the quantized radiation field, and there is no splitting due to relativistic effects.

Acknowledgments. We are grateful to Elliott Lieb for helpful discussions. C.H. was supported by a Marie Curie Fellowship of the European Community programme "Improving Human Research Potential and the Socioeconomic Knowledge Base" under contract number HPMFCT-2000-00660 and by the Deutsche Forschungsgemeinschaft, and acknowledges kind hospitality at Princeton University, where part of this work was done. R.S. was supported by the Austrian Science Fund in the form of an Erwin Schrödinger Fellowship.

\section{References}

[AF] C.-K. Au, G. Feinberg, Effects of retardation on electromagnetic selfenergy of atomic states, Phys. Rev. A 9, 1794-1800 (1974). Addendum ibid. 12, 1722 (1975). Erratum ibid. 12, 1733 (1975). 
[BFS] V. Bach, J. Fröhlich, I.-M. Sigal, Spectral Analysis for Systems of Atoms and Molecules Coupled to the Quantized Radiation Field, Commun. Math. Phys. 207, 249-290 (1999).

[Be] H.A. Bethe, The Electromagnetic Shift of Energy Levels, Phys. Rev. 72, 339-341 (1947).

[C] T. Chen, Operator-theoretic infrared renormalization and construction of dressed 1-particle states in non-relativistic $Q E D, \mathrm{PhD}$ thesis, ETH Zürich (2001). preprint arXiv:math-ph/0108021.

[D] M. Dresden, H.A. Kramers. Between Tradition and Revolution, Springer (1987).

[GLL] M. Griesemer, E.H. Lieb, M. Loss, Ground states in non-relativistic quantum electrodynamics, Inventiones Math. 145, 557-595 (2001).

[Gr] H. Grotch, Lamb shift in nonrelativistic quantum electrodynamics, Am. J. Phys. 49, 48-51 (1981). Erratum ibid. 49, 699 (1981).

[H] Ch. Hainzl, One non-relativistic particle coupled to a photon field, preprint arXiv:math-ph/0202001.

[HVV] Ch. Hainzl, V. Vougalter, S.A. Vugalter, Enhanced Binding in NonRelativistic QED, Commun. Math. Phys. (in press), preprint mp_arc $01-455$.

[Hi] F. Hiroshima, Self-adjointness of the Pauli-Fierz Hamiltonian for arbitrary values of coupling constants, Ann. Henri Poincaré 3, 171201 (2002).

[K] T. Kato, Perturbation theory for linear operator, Springer (1966).

[Ki] T. Kinoshita, The fine structure constant, Rep. Prog. Phys. 59, 145992 (1996).

[KL] N.M. Kroll, W.E. Lamb, On the Self-Energy of a Bound Electron, Phys. Rev. 75, 388-398 (1949).

[LL1] E.H. Lieb, M. Loss, Self-Energy of Electrons in non-perturbative $Q E D$, in: Differential Equations and Mathematical Physics, University of Alabama, Birmingham, 1999, R. Weikard and G. Weinstein, eds., 279-293, Amer. Math. Soc./Internat. Press (2000).

[LL2] E.H. Lieb, M. Loss, A Bound on Binding Energies and Mass Renormalization in Models of Quantum Electrodynamics, J. Stat. Phys. 108, 1057-1069 (2002). 